Essai sur le Développement de la Théorie des Fonctions de Variables Complexes. By Gaston Julia. Paris, Gauthier-Villars, 1933. viii +53 pp.

This pamphlet contains the lecture delivered by the author at the International Mathematical Congress at Zurich (1932). It covers a wide field, no less than that of the evolution of the subject from the time of Cauchy to the present, including the most recent work on quasi-analytic functions. The author, from the beginning, sets himself the task not only of outlining the main directions of progress in the theory of functions proper, but also of tracing the relations of its development to that of other mathematical disciplines.

A chapter is devoted to each of three periods. The first extends from the beginning of Cauchy's work to 1879, the year of Picard's theorem. The next goes on to 1900 , when the theory of point sets and of functions of real variables began to exercise a strong influence. This second period is marked by the dominance of the French school, including such names as those of Picard, Poincaré, Painlevé, Hadamard. The third period, from 1900 to the present, is sketched rapidly, but the author succeeds in giving some idea of the many modern lines of development. To indicate the amount of ground covered, the reviewer counted the names of seventy-four mathematicians whose work was cited in this last section. Twenty-eight of these were German, seventeen French, eleven Scandinavian, nine from eastern Europe, five Italian, and but two English and two native American. Does this represent a true appraisal of the importance of the various modern schools? The author would probably be the first to deny any such significance for his list of references; in fact he does so in his preface. But one looks in vain for any reference to the applications of this theory in the work of Birkhoff, for example. Perhaps it is too much to expect that a lecture of this length be encyclopedic.

D. R. Curtiss

Les Espaces de Finsler. By E. Cartan. (Actualités Scientifiques et Industrielles, No. 79.) Paris, Hermann, 1934. 40 pp.

In this pamphlet the author presents the recent developments in the geometry of Finsler spaces. He proposes five postulates for measurements in the space and by means of them obtains his tensor calculus. As he points out, his affine connection is essentially different from that of Berwald and it is more nearly analogous to that of a Riemann space; its main advantage is in the fact that lengths are preserved in parallel displacement. Of course here, as is usual, only spaces leading to a regular problem in the calculus of variations are considered. Of the scalar differential forms the author considers only the most important one - the angular metric of Landsberg, and he shows that it has curvature +1 . There are three sections on the geometry of curves and surfaces in a three-dimensional Finsler space which show to what extent or with what modifications classical differential geometry can be carried over to apply to Finsler spaces.

The author also considers some special $n$-dimensional spaces characterized by the vanishing of some tensor invariant; of particular interest is the one for which the determinant of the fundamental tensor is a point-function $\left(A_{i k^{k}}=0\right)$. Another interesting problem considered is the representation of the geometry 
of line elements (éléments d'appui) as the geometry of surface elements. The whole seems to be very closely related to the study of conservative dynamical systems as treated by contact transformations.

The pamphlet as a whole is extremely well done. The formulas are usually given geometrical content-an attribute often lacking in works on tensor analysis-and the proofs are clear and not too formal. Above all, brief as this pamphlet is, it contains many interesting ideas that seem to be worth elaborating.

\section{S. KNeBelman}

Gesammelte Abhandlungen. By David Hilbert. Zweiter Band. Algebra, Invariantentheorie, Geometrie. Berlin, Julius Springer, 1933.453+8 pp.

Turning over the leaves of the new volume of Hilbert's mathematical papers and comparing these theories more than forty years old with our present point of view brings again a vivid realization of the tremendous influence of Hilbert on the trends of mathematical thought. The investigations on invariant theory and algebraic systems form the main part of the second volume leading from his thesis (1885) to the two fundamental papers Über die Theorie der algebraischen Formen, Mathematische Annalen vol. 36 (1890) and Über die vollen Invariantensysteme, ibid., vol. 42 (1893). In a short expository article van der Waerden gives an account of the continuation of Hilbert's work on algebraic systems through Lasker and Macaulay up to the abstract formulation by $\mathrm{E}$. Noether's ideal theory in commutative rings.

Among other papers of great importance one should mention paper No. 18 containing Hilbert's irreducibility theorem and the investigations on equations with a prescribed Galois group and also Nos. 10 and 20 dealing with the representation of definite forms as the sum of squares, a problem which was first completeiy solved by Artin. Paper 26 contains the results on the still unsolved problem of representing the solution of a general algebraic equation by means of functions of a minimal number of variables.

The geometric part of the works is incompletely representative since the well known book Grundlagen der Geometrie could not be included. This lacuna is partly filled by an interesting article by Arnold Schmidt on the recent contributions to the axiomatics of euclidean geometry.

OysteIN ORE

Grundbegriffe der Wahrscheinlichkeitsrechnung. By A. Kolmogoroff. Berlin, Julius Springer, 1933. $62 \mathrm{pp}$.

It is the purpose of this monograph to develop probability theory from a postulational standpoint. For this purpose a probability field is defined as an assemblage with a definite ordering of numbers that satisfy the system of axioms. A brief exposition is given of the construction of such fields and of the manner in which the framework of the postulational system can be related to the applications to phenomena. The addition and multiplication theorems follow at once. Moreover, the theorem of Bayes, concerning whose validity there have been many controversies, is also an almost immediate consequence of the system of postulates, but the reviewer does not think this derivation of 